\title{
ACCESIBILIDAD Y CONECTIVIDAD GEOGRÁFICA EN ÁREAS RURALES. CASO DE LA COMUNA DE MARÍA PINTO, CHILE
}

\author{
Gerardo Ubilla-Bravo \\ Institut National de Recherche Agronomique (Francia) y Université Paul-Valéry (Francia)
}

\begin{abstract}
RESUMEN
En América Latina y especialmente en Chile, las áreas rurales son espacios que todavía presentan brechas de desarrollo en torno a la accesibilidad y la conectividad geográfica del territorio. La geografía puede aportar enfoques conceptuales y metodológicos para superar las brechas espaciales.Este artículo apunta a determinar y categorizar la accesibilidad y conectividad geográfica que tiene la población rural respecto de su centro urbano. Consideramos el estudio de caso como enfoque para analizar este tema, abordando de este modo la situación de la comuna de María Pinto, localizada en la Región Metropolitana de Santiago, Chile. Los objetivos que guían esta investigación son: caracterizar la red vial existente, determinar la conectividad y la accesibilidad geográfica intracomunal y determinar áreas homogéneas de accesibilidad en relación con el centro urbano de la comuna de María Pinto. En el método se usan los índices de Konig y de Shimbel para la conectividad, el índice de Rodeo para la accesibilidad y las superficies de fricción mediante una fórmula para calcular las áreas en isócronas. En la discusión y conclusiones planteamos la relevancia que estos conceptos tienen con la movilidad de la población rural como parte de las políticas de desarrollo.
\end{abstract}

Palabras clave: accesibilidad geográfica; conectividad geográfica; área rural; María Pinto; Chile.

\section{ACCESSIBILITY AND GEOGRAPHICAL CONNECTIVITY IN RURAL AREAS. CASE OF THE COMMUNE MARIA PINTO, CHILE}

\section{ABSTRACT}

In Latin America and especially in Chile, rural areas are spaces that still have developmental gaps around the accessibility and geographical connectivity of the territory. Geography can provide conceptual and methodological approaches to overcome spatial gaps. This article aims to determine and categorize the accessibility and geographical connectivity that the rural population has in relation to its urban center. We consider the case study as an approach to analyze this issue, thus addressing the situation of the municipality of María Pinto, located in the Metropolitan Region of Santiago, Chile. The objectives that guide this research are: characterize the existing road network, determine connectivity and intracommunal geographical accessibility and determine homogeneous areas of accessibility regarding the urban center of the commune of Maria Pinto. In the method, the Konig and Shimbel indexes are used for connectivity, the Rodeo index for accessibility and friction surfaces using a formula to calculate the areas in isochronous. In the discussion and conclusions we discuss the relevance of these concepts to the mobility of the rural population as part of development policies.

Key words: geographic accessibility; geographic connectivity; rural area; María Pinto; Chile.

\footnotetext{
${ }^{1}$ Université Paul-Valéry, Montpellier III. E-mail: gerardo.ubilla_bravo@yahoo.fr
}

Fecha de recepción: 21 de julio de 2017. Fecha de aceptación: 16 de octubre de 2017. 


\section{INTRODUCCIÓN}

\subsection{CONSIDERACIONES CONCEPTUALES EN TORNO A LA ACCESIBILIDAD Y CONECTIVIDAD GEOGRÁFICA}

La accesibilidad y la conectividad son temas de particular interés para la geografía como disciplina científica. Comprender la dinámica de estructuración de los desplazamientos de la población en el espacio geográfico es una interrogante planteada por varios autores a lo largo de varias décadas (ESCALONA ORCAO et al., 2003; GÓMEZ FAYRÉN et al., 1990; FARROW et al., 2001; PIRIE, 1979; SHIMBEL, 1953; WEBER, 2006). En general, el principal enfoque para examinar los fenómenos asociados a estos conceptos son los flujos de viajes por estudio y por trabajo (MORRISet al., 1979; WEBER, 2006), y su examen es relevante e útil para los estudios asociados tanto al transporte (PÁEZ et al., 2012) como a la localización de servicios y actividades urbanas (MONIRUZZAMAN et al., 2017).

En relación con su definición, la accesibilidad geográfica es un concepto que ha tenido varias interpretaciones y definiciones, y a menudo se usa para facilitar comparaciones temporales o espaciales (MORRIS et al., 1979). Para PÁEZ et al., (2012) la accesibilidad es el potencial para alcanzar o conseguir oportunidades que se distribuyen en el espacio. Estas oportunidades son elementos que ofrecen un servicio cuya localización es fija. También es considerado como la facilidad con la que un lugar atractivo (desde un punto de vista económico) alcanza a otros lugares (BAVOUX et al., 2009). Otra definición indica que la accesibilidad geográfica es una distancia que permite comprender la proximidad, la separación y/o la discontinuidad entre dos o más elementos en el espacio (FERREIRA et al., 2013). También puede ser considerada como una medida de separación espacial entre actividades humanas (MORRIS et al., 1979), donde existe un centro proveedor de un servicio o empleo y su espacio circundante (MONIRUZZAMANet al., 2017). FERREIRA et al., (2012) en concordancia con GUAGLIARDO (2004) proponen cuatro tipos de orientaciones para abordar el análisis espacial de la accesibilidad: (i) la proporción entre el proveedor de un servicio y la población, (ii) la distancia hacia el proveedor de un servicio, (iii) la distancia media hacia un conjunto de proveedores de un servicio y (iv) los modelos de gravitación espacial.

Pasando a la conectividad geográfica, AUNTA PEÑA(2014) plantea que es la identificación de atributos naturales y artificiales que determinan la integración de un territorio. Desde el punto de vista metodológico, este autor indica que la conectividad sirve como categoría de análisis para la planificación regional (AUNTA PEÑA 2014).BAVOUX et al. (2009) plantean que la conectividad es una propiedad de una red para ofrecer itinerarios alternativos entre los centros.ITONGWA et al.(2002) y DUPUY (1985)sostienen que la conectividad es el grado de cohesión entre los distintos centros de una red de vías. Dichos centros tienen una jerarquía que determinan los niveles de conexión (MUÑIZ SOLARI, 1985). En este sentido la conectividad debe ser entendida como un sistema dinámico y no como elementos sin interacción. Esta noción se adapta a nuestra visión sobre la conectividad intracomunal que desarrollaremos en este estudio. Por otra parte, ESPINOZA LIZAMA(2010) aporta señalando la relevancia de la conectividad como indicador a ser considerado para la medición de desarrollo local y en el establecimiento de áreas de planificación.

\subsection{PROBLEMÁTICA: LA ACCESIBILIDAD Y CONECTIVIDAD COMO POLÍTICA PÚBLICA EN LA PLANIFICACIÓN TERRITORIAL}

El acceso de la población a los servicios públicos básicos es parte de los componentes del bienestar(ESCALONA ORCAO et al., 2003). De esta forma, tanto la accesibilidad como la conectividad geográfica cobran relevancia si se consideran en la planificación territorial y la formulación de políticas públicas (YOSHIDA et al., 2009). Esto da paso tanto a la construcción de vías y caminos como al análisis del transporte público que pueda llegar a dichas áreas (GÓMEZ FAYRÉNet al., 1990). Uno de sus objetivos es proveer las condiciones para mejorar la calidad de la accesibilidad, sobre todo en los países del sur (RUSHTON,1984). En este sentido,ITONGWA et al., (2002) confirman el argumento anterior indicando que el interés de 
este tipo de estudios contribuye al ordenamiento territorial de naciones en vías de desarrollo. Esto tiene relación con lo que sucede en América Latina y especialmente en Chile, donde las áreas rurales son espacios que todavía presentan brechas de desplazamiento en torno a la accesibilidad y la conectividad geográfica del territorio (UBILLA BRAVO 2012; UBILLA BRAVO et al., 2014; SALAZAR et al., 2014). En general las áreas rurales presentan una baja densidad de población y existen pocos servicios que cubran necesidades de servicios como la educación o la salud (ESCALONA ORCAO et al., 2003).

Pasando a la problemática particular de esta investigación señalamos dos puntos. Primero, que en Chile existe poca producción académica desde la geografía en torno a los temas de la accesibilidad y conectividad geográfica en áreas rurales. Estas investigaciones se concentran más bien en el análisis en áreas urbanas, preferentemente metropolitanas. De esta manera queremos construir con el conocimiento de cómo se comporta el espacio en relación con el tiempo de desplazamientos de habitantes que viven en un medio rural. En particular queremos conocer los desplazamientos que deben hacer desde distintos lugares a su cabecera comunal, asentamiento que provee de servicios a la población rural.En segundo lugar, constatamos que todavía persisten brechas en cuanto al acceso a servicios básicos de la población que habita en comunas rurales.

Inspirándonos en el trabajo realizado por GÓMEZ FAYRÉN et al., (1990) para la Región de Murcia en España, los objetivos que guían esta investigación son: (i) caracterizar la red vial existente (carpeta, longitud, distribución), (ii) determinar la conectividad geográfica intracomunal mediante los índices de Konig y Shimbel, (iii) determinar la accesibilidad geográfica intracomunal según el índice de rodeo y (iv) determinar áreas homogéneas de accesibilidad mediante isócronas en relación con el centro urbano de la comuna de María Pinto.

\section{MATERIALES Y MÉTODOS}

\section{1.ÁREA DE ESTUDIO, RED VIAL Y DETERMINACIÓN DE LA CONECTIVIDAD GEOGRÁFICA ENTRE ASENTAMIENTOS}

El área de estudio elegido para realizar este análisis corresponde a la comuna de María Pinto, localizada en la Región Metropolitana de Santiago (ver FIGURA 1). Esta se encuentra a $65 \mathrm{~km}$ al oeste de la ciudad de Santiago, en la zona central del valle del estero Puangue y contiene cerca de 11.000 según el Instituto Nacional de Estadística (INE) de Chile. La apuesta en este trabajo es mostrar la diferenciación espacial en la accesibilidad y conectividad geográfica de una comuna que es de carácter rural, pero que se encuentra en una región que contiene un área metropolitana.Con esto demostramos que estos fenómenos están presentes en cualquier tipo de región en Chile.

El primer paso metodológico consistió en calcular la longitud de todas las vías que se encuentran en la comuna, tal como sugieren GÓMEZ FAYRÉN et al., (1990). En segundo lugar, se consideraron tres categorías según la carpeta de las vías: pavimento (incluyendo asfalto), ripio y tierra. De esta forma se puede determinar las distintas velocidades de desplazamiento (FERREIRA et al., 2013). Posteriormente ubicamos los centroides de todos los asentamientos humanos de la comuna, los que servirán como nodos en el análisis. La fuente de estos datos georreferenciados es el Instituto Geográfico Militar (IGM) de Chile los que fueron actualizados por el Gobierno Regional Metropolitano de Santiago (GORE RMS). Además, hemos utilizado el software QGIS para la representación, análisis y producción de los datos georreferenciados. Una vez que todos los asentamientos humanos (nodos) de la comuna de María Pinto fueron localizados con todas las vías (arcos) que unen a estos, procedemos a aplicar los índices de conectividad.

De este modo, el segundo paso es asignar a cada nodo el valor de arcos en relación con cada uno de los otros nodos. La conectividad geográfica intracomunal la medimos mediante los índices de Konig y de Shimbel. El índice de Konig consiste en el valor o cantidad de arcos más alto en relación con el nodo más lejano (mayor cantidad de arcos) (PIRIE, 1979; GRUBESIC et al., 2008; CÉSPEDES ÁLVAREZ, 2016; DEL CANTO FRESNO et al., 1998). El índice de Shimbel es la sumatoria de todos los arcos que tiene un nodo en relación con los demás 
(SHIMBEL, 1953; ITONGWA et al., 2002).

FIGURA 1

Contexto geográfico del área de estudio, comuna de María Pinto

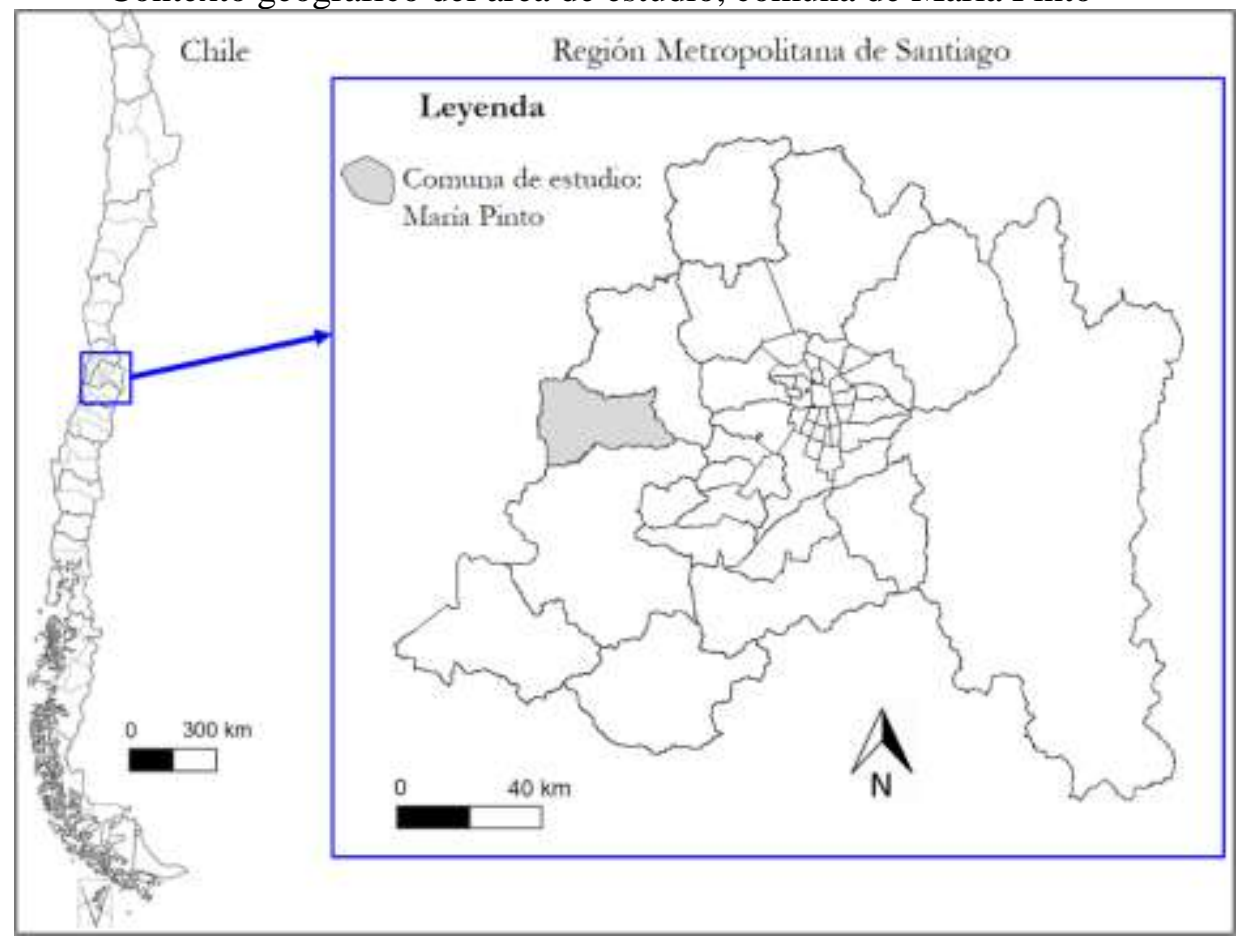

Fuente: Elaboración propia a partir de datos georreferenciados por el Gobierno Regional Metropolitano de Santiago.

\subsection{DETERMINACIÓN DE LA ACCESIBILIDAD GEOGRÁFICA ENTRE ASENTAMIENTOS}

Como mencionamos anteriormente, la conectividad se basó en un modelo de nodos y arcos. Para la accesibilidad geográfica entre asentamientos continuamos usando ese modelo como base. La diferencia estriba que en lugar de medir arcos como un valor, en este objetivo calculamos dos tipos de distancias entre centros poblados. La distancia real que corresponde a los km de las vías entre cada asentamiento y la distancia ideal que es una línea recta (distancia mínima) entre dos nodos, también medido en $\mathrm{km}$. Estos datos servirán de entrada para calcular el Índice de Rodeo (IdR) (DEL CANTO FRESNO et al., 1998) o de Trazado (SEGUÍ PONS et al., 1991). Este consiste en dividir los valores de la distancia real con la ideal (o mínima) en relación con dos centros poblados o con el total (dividiendo las sumatorias) (ANDRADE GARCÍAet al., 2008; GÓMEZ FAYRÉNet al., 1990). A continuación señalamos como se deben interpretar los datos. Si los valores se acercan a ' 1,00 ' la distancia real es poco mayor que la ideal, y si es igual a ' 1,00 ', significa que es la misma distancia (la real e ideal), en ambos es una alta accesibilidad. Por el contrario, mientras más se aleje el valor a ' 1,00 ', entonces la distancia real será mucho mayor respecto de la ideal(DEL CANTO FRESNO et al., 1998).

\subsection{DETERMINACIÓN DE LAS UNIDADES HOMOGÉNEAS DE ACCESIBILIDAD GEOGRÁFICA}

La determinación de las unidades homogéneas de accesibilidad geográfica se desarrolla a partir de la aplicación de un modelo de costo de superficie acumulada(DOUGLAS, 1994) que considera los siguientes puntos:

- Pasar a formato raster (cuadrícula) todos los caminos ya vectorizados de la comuna. La cuadrícula del raster representa un valor de cinco por cinco metros. 
- Dar un valor 'cero' a la cuadrícula por donde pasa un camino y aplicar un buffer desde el camino. Esto significa que todos los espacios fuera de los caminos van a tener un valor de distancia respecto al camino. De este modo, las zonas adyacentes a los caminos tendrán un valor más cercano a cero porque es más próximo al camino, mientras que los espacios más alejados tendrán un valor más alto. El valor corresponde a la distancia en metros. El resultado de esto es un mapa de distancias euclidianas a partir de los caminos.

- Realizar un modelo digital de terreno a partir de datos georreferenciados con curvas de nivel y calcular su gradiente de pendiente, tal como lo desarrolla en su investigación CÉSPEDES ÁLVAREZ (2016).

- Aplicar el mapa de gradiente de pendiente al mapa de las distancias euclidianas. De esta forma, las distancias estarán en razón de los desniveles del terreno, y no estarán en plano (geometría euclidiana). La fórmula de la FIGURA 2 fue aplicada al mapa euclidiano para realizar el mapa de distancias que consideran la pendiente. El propósito de esto es considerar en el análisis las formas del relieve para que los resultados expresen de mejor forma el comportamiento de este fenómeno en el territorio local (ITONGWAet al., 2002).

FIGURA 2

i) Triángulo rectángulo con sus propiedades trigonométricas, ii) fórmula de cálculo gradiente de la pendiente

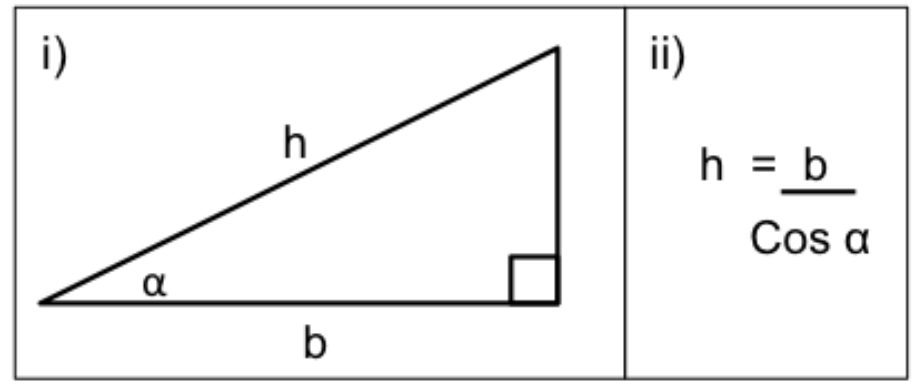

Fuente: Elaboración propia. * Explicación de letras de i): "h": hipotenusa (distancia real), "b": cateto adyacente (distancia euclidiana), " $\alpha$ ": ángulo alfa (gradiente de pendiente).

- Al mapa de distancias que considera la gradiente de pendiente se le aplicaron valores de superficies de fricción al interior de la comuna (TABLA 1 y TABLA 2). Estos valores fueron obtenidos en un trabajo de toma de puntos y distancias realizada en terreno. Posteriormente vemos por separado el desplazamiento a través de las vías en vehículo (que considera el tipo de carpeta) y a pie (esta última considera la vegetación como fricción) como sugieren TANSER et al.,(2006). El detalle de los valores por tipo de carpeta cobertura cruzado con las gradientes de pendientes se encuentran en la TABLA 1 y TABLA 2 .

TABLA 1

Valores en las respectivas superficies de fricción para las vías en vehículo, según tipo de carpeta $\mathrm{y}$ gradiente de pendiente (en $\mathrm{km} / \mathrm{hr}$ )

\begin{tabular}{lcccc}
\hline $\begin{array}{c}\text { Gradiente de pendiente } \\
\text { Tipo de carpeta }\end{array}$ & $\begin{array}{c}\text { Plano } 0^{\circ}-5^{\circ} \\
\mathrm{km} / \mathrm{hr}\end{array}$ & $\begin{array}{c}5^{\circ}-10^{\circ} \\
\mathrm{km} / \mathrm{hr}\end{array}$ & $\begin{array}{c}10^{\circ}-20^{\circ} \\
\mathrm{km} / \mathrm{hr}\end{array}$ & $\begin{array}{c}20^{\circ} \mathrm{y} \text { más } \\
\mathrm{km} / \mathrm{hr}\end{array}$ \\
\hline Pavimento & 50 & 45 & 40 & 35 \\
Ripio & 30 & 25 & 20 & 15 \\
Tierra & 20 & 15 & 10 & 8 \\
\hline
\end{tabular}

Fuente: Elaboración propia, a partir de datos obtenidos en terreno. 
TABLA 2

Valores en las respectivas superficies de fricción para el desplazamiento a pie, según la cobertura vegetal y el gradiente de pendiente (en $\mathrm{km} / \mathrm{hr}$ )

\begin{tabular}{lcccc}
\hline \multicolumn{1}{c}{$\begin{array}{c}\text { Gradiente de pendiente } \\
\begin{array}{l}\text { Tipo de cobertura del } \\
\text { suelo }\end{array}\end{array}$} & $\begin{array}{c}\text { Plano } 0^{\circ}-5^{\circ} \\
\mathrm{km} / \mathrm{hr}\end{array}$ & $\begin{array}{c}5^{\circ}-10^{\circ} \\
\mathrm{km} / \mathrm{hr}\end{array}$ & $\begin{array}{c}10^{\circ}-20^{\circ} \\
\mathrm{km} / \mathrm{hr}\end{array}$ & $\begin{array}{c}20^{\circ} \mathrm{y} \mathrm{más} \\
\mathrm{km} / \mathrm{hr}\end{array}$ \\
\hline $\begin{array}{l}\text { Sin Vegetación } \\
\text { Con Vegetación (matorral }\end{array}$ & 5,0 & 4,5 & 4,0 & 3,5 \\
semi-abierto) & 3,0 & 2,5 & 2,0 & 1,5 \\
\hline
\end{tabular}

Fuente: Elaboración propia, a partir de datos obtenidos en terreno.

A partir de los valores detallados en la TABLA 1 y TABLA 2 se aplicó una fórmula del modelo matemático de accesibilidad (ver FIGURA 3) propuesto por FARROW et al., (2001) para el Centro Internacional de Agricultura Tropical (CIAT).

FIGURA 3

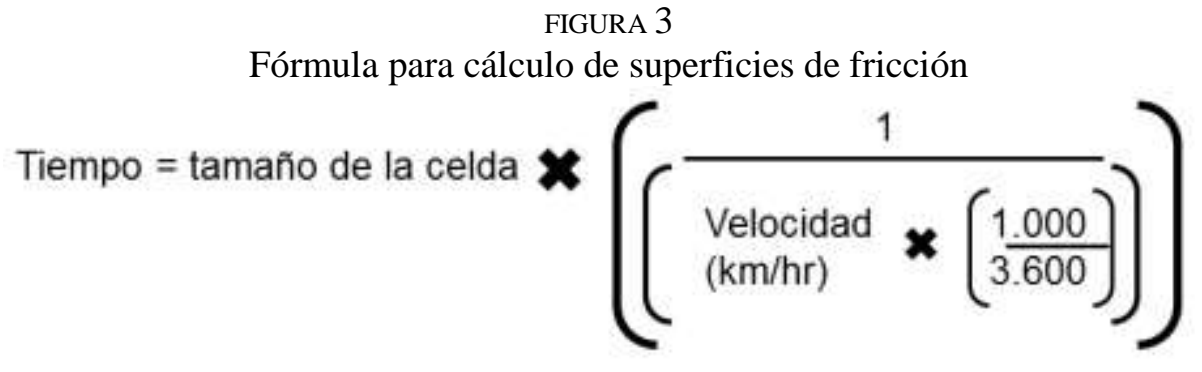

Fuente: Elaboración propia.

Siguiendo las investigaciones de varios autores (MONIRUZZAMAN et al., 2017; ESCALONA ORCAO et al., 2003; FERREIRA et al., 2013), el producto final de todo este trabajo es el mapa accesibilidad por isocronas que tiene cualquier localización de la comuna hacia el centro poblado de María Pinto, cuyo análisis será tratado en la sección de resultados. Si bien la fórmula de superficies de fricción (FIGURA 3) se expresa en segundos, hemos transformado los valores en minutos para facilitar la lectura de los resultados. La determinación de las unidades homogéneas consistió en la categorización del mapa anterior en cuatro tipos: (i) alta accesibilidad: menor a 30 minutos, (ii) media accesibilidad: entre 30 y 60 minutos, (iii) baja accesibilidad: entre 60 y 120 minutos y (iv) muy baja accesibilidad: más de 120 minutos (dos horas). Finalmente se calcula el área de cada una de estas tipologías para poner en evidencia las brechas de accesibilidad intracomunal.

\section{RESULTADOS}

\subsection{CARACTERIZACIÓN DE LA RED VIALDE MARÍA PINTO}

Los valores de longitud $(\mathrm{km})$ y el porcentaje de la cobertura y cantidad de los caminos se observan en latabla 3. La mayoría de los caminos de la comuna de María Pinto son de tierra y conforman casi el 60\% según los datos producidos por el GORE RMS. Enseguida apreciamos que los caminos con carpeta de ripio representan un $24 \%$ del total, siendo los más rápidos para pavimentar. Finalmente, los caminos pavimentados donde la velocidad del desplazamiento es mayor representan un $16 \%$. 
TABLA 3

Valores en las respectivas superficies de fricción para el desplazamiento a pie, según la cobertura vegetal y el gradiente de pendiente (en $\mathrm{km} / \mathrm{hr}$ )

\begin{tabular}{ccc}
\hline Tipo de Carpeta & Longitud $(\mathrm{km})$ & Proporción $(\%)$ \\
\hline Tierra & 248,14 & 59,34 \\
Ripio & 101,74 & 24,33 \\
Pavimentado & 68,26 & 16,32 \\
Total & 418,14 & 100,00
\end{tabular}

Fuente: Elaboración propia a partir de datos georreferenciados del GORE RMS.

Dos elementos se aprecian en la FIGURA 4: la localización de los centros poblados y las vías de María Pinto. En cuanto a esta última, sus disparidades espaciales quedan en evidencia en la comuna. La disposición asimétrica de la red vial en el territorio comunal se explica en parte por su relieve. La red de los caminos de tierra se presenta hacia la periferia, la que corresponde a aquellos lugares de media montaña: laderas con pendientes abruptas que corresponden a la cordillera de la Costa. Mientras que los caminos pavimentados sólo se encuentran en la zona de valle y representan a las vías principales de acceso. El patrón espacial de las vías con ripio no es simétrico y se ubican indistintamente en zonas de valle y media montaña.

FIGURA 4

Distribución espacial de los centros poblados y los caminos en la comuna de María Pinto

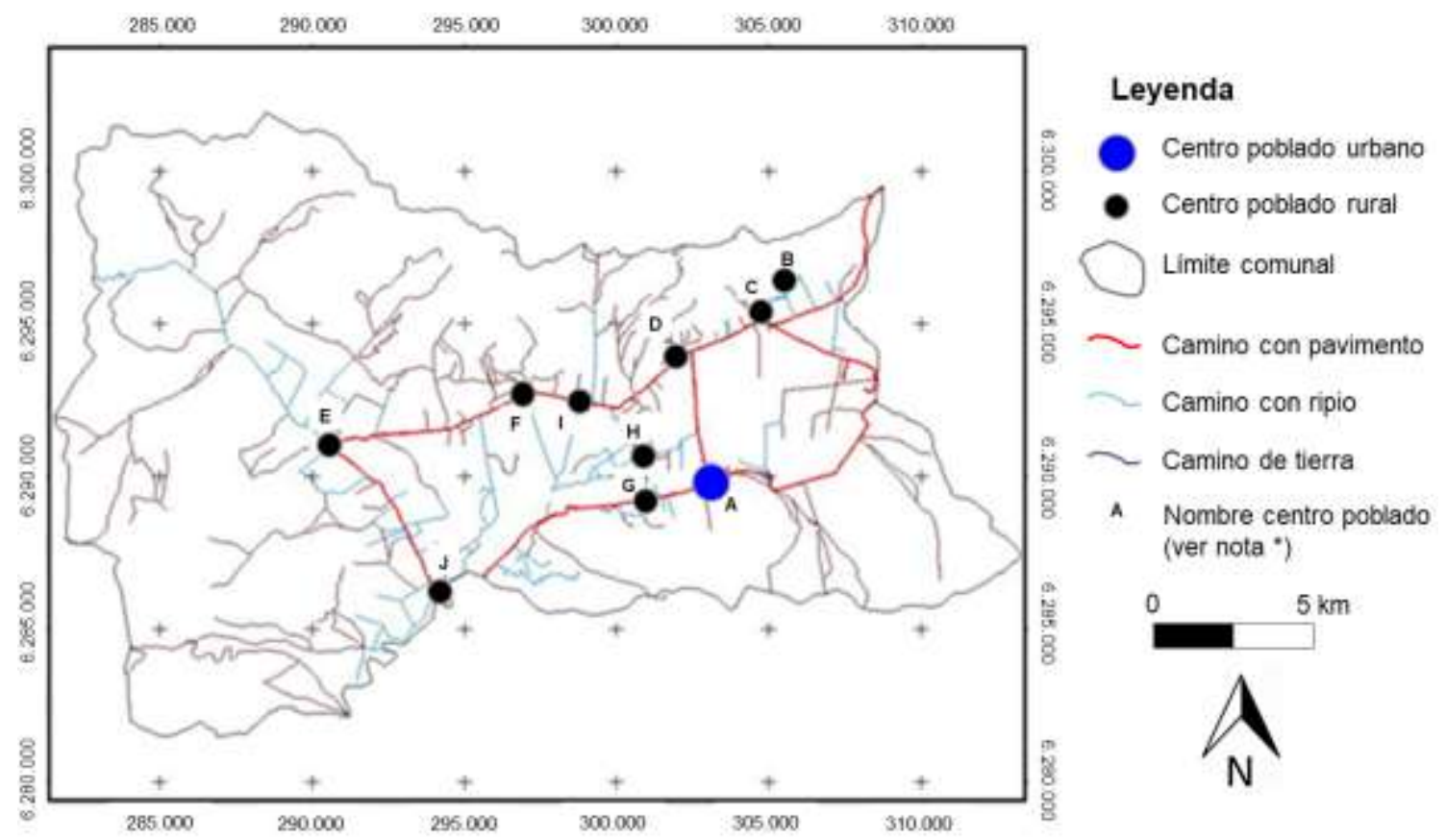

Fuente: Elaboración propia. * Nombre de los centros poblados: María Pinto (A), Villa Santa Luisa (B), Santa Emilia (C), La Estrella (D), Chorombo Alto (E), Las Mercedes (F), Baracaldo (G), El Rosario (H), Malalhue (I), Chorombo Bajo (J).

\subsection{CONECTIVIDAD GEOGRÁFICA INTRACOMUNAL ENTRE ASENTAMIENTOS}

En cuanto a la conectividad intracomunal entre asentamientos (o centros poblados), en la TABLA 4 se aprecian los valores para los diez asentamientos indicados en la zona inferior de la FIGURA 4. Tal como se señaló en el método, los valores corresponden a los arcos entre cada uno de los nodos, lo que proporciona una mirada general de la conectividad geográfica intracomunal. En este sentido, los centros poblados Malalhue (I) y La Estrella (D) son los que están más conectados según el índice de Konig (valor igual a 4). El caso se repite con el índice de Shimbel para La Estrella (D) teniendo un valor de 21. En cuanto a la situación contraria, 
Villa Santa Luisa (B) y Chorombo Bajo presentan los valores más altos índice de conectividad geográfica respecto de los otros centros poblados. Si tomamos en consideración el índice de Shimbel, Villa Santa Luisa (B) es por lejos el más alto con un valor de 41.

TABLA 4

Matriz de conectividad para los diez asentamientos poblados en la comuna de María Pinto

\begin{tabular}{lcccccccccccl}
\hline \multicolumn{1}{c}{ Centro poblado } & A & B & C & D & E & F & G & H & I & J & Konig & Shimbel \\
\hline María Pinto (A) & - & 4 & 2 & 2 & 5 & 4 & 1 & 2 & 3 & 3 & 5 & 26 \\
Villa Santa Luisa (B) & 4 & - & 2 & 3 & 6 & 5 & 5 & 5 & 4 & 7 & 7 & 41 \\
Santa Emilia (C) & 2 & 2 & - & 1 & 4 & 3 & 3 & 3 & 2 & 5 & 5 & 25 \\
La Estrella (D) & 2 & 3 & 1 & - & 3 & 2 & 3 & 2 & 1 & 4 & 4 & 21 \\
Chorombo Alto (E) & 5 & 6 & 4 & 3 & - & 1 & 3 & 4 & 2 & 1 & 6 & 29 \\
Las Mercedes (F) & 4 & 5 & 3 & 2 & 1 & - & 4 & 3 & 1 & 2 & 5 & 25 \\
Baracaldo (G) & 1 & 5 & 3 & 3 & 3 & 4 & - & 3 & 4 & 2 & 5 & 28 \\
El Rosario (H) & 2 & 5 & 3 & 2 & 4 & 3 & 3 & - & 2 & 5 & 5 & 29 \\
Malalhue (I) & 3 & 4 & 2 & 1 & 2 & 1 & 4 & 2 & - & 3 & 4 & 22 \\
Chorombo Bajo (J) & 3 & 7 & 5 & 4 & 1 & 2 & 2 & 5 & 3 & - & 7 & 32 \\
\hline
\end{tabular}

Fuente: Elaboración propia.

\subsection{ACCESIBILIDAD GEOGRÁFICA INTRACOMUNAL ENTRE ASENTAMIENTOS}

Para la accesibilidad geográfica tenemos tres tipos de resultados a partir de la red de caminos: la accesibilidad real (AcR), la accesibilidad ideal (AcI) y el índice de rodeo (IRd). Los resultados de los primeros dos casos se advierten en la TABLA 5 y TABLA 6cuyos valores corresponden a $\mathrm{km}$. En el caso de la distancia real, los centros poblados Chorombo Alto (E) y Chormbo Bajo (J) son los que está a mayor distancia de todos los centros poblados. La razón que explica estos valores corresponde a su localización, ya que se encuentran en una zona periférica respecto de las otras localidades, cerca de la zona de pendientes. El asentamiento El Rosario $(\mathrm{H})$ es el que presenta menor distancia de los otros asentamientos. Esto se debe que está en el centro de la comuna, cerca del centro urbano como se aprecia en la FIGURA 4.

TABLA 5

Accesibilidad real (AcR) para asentamientos poblados en la comuna de María Pinto (longitud en

\begin{tabular}{cccccccccccc}
\multicolumn{1}{c}{$\begin{array}{c}\text { Centro } \\
\text { Poblado }\end{array}$} & A & B & C & D & E & F & G & H & I & J & AcR \\
\hline A & 0,00 & 7,78 & 6,30 & 4,10 & 14,74 & 9,41 & 1,29 & 3,19 & 7,95 & 9,29 & 64,05 \\
B & 7,78 & 0,00 & 1,43 & 4,48 & 20,24 & 13,78 & 9,15 & 9,18 & 8,33 & 17,07 & 91,44 \\
C & 6,30 & 1,43 & 0,00 & 3,00 & 14,97 & 8,31 & 7,59 & 7,61 & 6,85 & 15,59 & 71,45 \\
D & 4,10 & 4,48 & 3,00 & 0,00 & 11,77 & 5,31 & 5,39 & 3,68 & 3,85 & 13,39 & 54,97 \\
E & 14,74 & 20,24 & 14,97 & 11,77 & 0,00 & 6,46 & 13,45 & 10,47 & 7,92 & 5,45 & 105,27 \\
F & 9,41 & 13,78 & 8,31 & 5,31 & 6,46 & 0,00 & 10,70 & 4,01 & 1,46 & 11,91 & 80,56 \\
G & 1,29 & 9,15 & 7,59 & 5,39 & 13,45 & 10,70 & 0,00 & 4,48 & 9,24 & 8,00 & 78,50 \\
H & 3,19 & 9,18 & 7,61 & 3,68 & 10,47 & 4,01 & 4,48 & 0,00 & 2,55 & 12,48 & 57,65 \\
I & 7,95 & 8,33 & 6,85 & 3,85 & 7,92 & 1,46 & 9,24 & 2,55 & 0,00 & 13,37 & 61,52 \\
J & 9,29 & 17,07 & 15,59 & 13,39 & 5,45 & 11,91 & 8,00 & 12,48 & 13,37 & 0,00 & 106,55 \\
\hline
\end{tabular}

Fuente: Elaboración propia. * Nombre de los centros poblados: María Pinto (A), Villa Santa Luisa (B), Santa Emilia (C), La Estrella (D), Chorombo Alto (E), Las Mercedes (F), Baracaldo (G), El Rosario (H), Malalhue (I), Chorombo Bajo (J).

La situación presentada en la accesibilidad real es similar a la accesibilidad ideal (AcI). Los tres centros poblados indicados anteriormente: Chorombo Alto (E) y Chormbo Bajo (J) y El 
Rosario $(\mathrm{H})$ son los mismos que muestran valores extremos. La diferencia que se puede observar en la TABLA 6entre ambas accesibilidades es que la ideal son menos km lo que se asocia al método utilizado. La relevancia de usar los dos índices es que permiten cotejar los valores de ambos casos y ver si existe una relación en el patrón geográfico de los nodos.

TABLA 6

Accesibilidad ideal (AcI) para asentamientos poblados en la comuna de María Pinto (longitud

\begin{tabular}{cccccccccccc}
$\begin{array}{l}\text { Centro } \\
\text { Poblado }\end{array}$ & A & B & C & D & E & F & G & H & I & J & AcI \\
\hline A & 0,00 & 5,69 & 4,70 & 3,55 & 12,05 & 6,04 & 1,23 & 1,85 & 4,34 & 8,81 & 48,26 \\
B & 5,69 & 0,00 & 0,77 & 3,82 & 15,05 & 8,69 & 7,89 & 6,87 & 7,26 & 14,74 & 70,78 \\
C & 4,70 & 0,77 & 0,00 & 2,91 & 14,26 & 7,85 & 6,75 & 5,83 & 6,38 & 13,70 & 63,15 \\
D & 3,55 & 3,82 & 2,91 & 0,00 & 10,75 & 4,35 & 4,34 & 2,72 & 2,82 & 10,31 & 45,57 \\
E & 12,05 & 15,05 & 14,26 & 10,75 & 0,00 & 5,96 & 9,91 & 9,45 & 7,62 & 5,09 & 90,14 \\
F & 6,04 & 8,69 & 7,85 & 4,35 & 5,96 & 0,00 & 4,86 & 3,77 & 1,44 & 6,56 & 49,52 \\
G & 1,23 & 7,89 & 6,75 & 4,34 & 9,91 & 4,86 & 0,00 & 1,17 & 3,51 & 6,99 & 46,65 \\
H & 1,85 & 6,87 & 5,83 & 2,72 & 9,45 & 3,77 & 1,17 & 0,00 & 2,07 & 7,47 & 41,20 \\
I & 4,34 & 7,26 & 6,38 & 2,82 & 7,62 & 1,44 & 3,51 & 2,07 & 0,00 & 7,19 & 42,63 \\
J & 8,81 & 14,74 & 13,70 & 10,31 & 5,09 & 6,56 & 6,99 & 7,47 & 7,19 & 0,00 & 80,86 \\
\hline
\end{tabular}

Fuente: Elaboración propia. * Nombre de los centros poblados: María Pinto (A), Villa Santa Luisa (B), Santa Emilia (C), La Estrella (D), Chorombo Alto (E), Las Mercedes (F), Baracaldo (G), El Rosario (H), Malalhue (I), Chorombo Bajo (J).

La TABLA 7 muestra el índice de rodeo (IRd) para los diez asentamientos humanos de la comuna de María Pinto. Recordamos que este valor es la división de la accesibilidad real (AcR)por la ideal (AcI) y no tiene necesariamente relación con los valores extremos de los otros dos índices ya analizados. De este modo se aprecia que el poblado Las Mercedes (F) tiene el valor más alto del IRd, esto quiere decir que es el asentamiento que presenta una mayor distancia (la más alejada del óptimo, distancia ideal), dada por la distribución de caminos que los unen a los otros asentamientos. Por el contrario, el centro que más se acerca al óptimo ' 1,00 ' es la localidad Chorombo Alto (E) con un valor 1,17. Este dato es interesante porque en el caso de las otras dos distancias analizadas (AcR y AcI) representa el valor más alto, pero aquí tiene el índice más bajo en conjunto. En cuanto a otros valores observamos un IRd 3,83 entre los asentamientos de Baracaldo $(\mathrm{G})$ y El Rosario (H) lo que se explica por su localización, ya que el camino no va en línea recta atravesando el estero Puangue si no que hay que hacer una vuelta pasando por la capital comunal (A)(apóyese en la FIGURA 4).

TABLA 7

Índice de rodeo (IRd) para los diez asentamientos poblados en la comuna de María Pinto

\begin{tabular}{cccccccccccc}
\hline $\begin{array}{l}\text { Centro } \\
\text { Poblado }\end{array}$ & A & B & C & D & E & F & G & H & I & J & IRd \\
\hline A & - & 1,37 & 1,34 & 1,15 & 1,22 & 1,56 & 1,05 & 1,72 & 1,83 & 1,05 & 1,33 \\
B & - & - & 1,86 & 1,17 & 1,34 & 1,59 & 1,16 & 1,34 & 1,15 & 1,16 & 1,29 \\
C & - & - & - & 1,03 & 1,05 & 1,06 & 1,12 & 1,31 & 1,07 & 1,14 & 1,13 \\
D & - & - & - & - & 1,09 & 1,22 & 1,24 & 1,35 & 1,37 & 1,30 & 1,21 \\
E & - & - & - & - & - & 1,08 & 1,36 & 1,11 & 1,04 & 1,07 & 1,17 \\
F & - & - & - & - & - & - & 2,20 & 1,06 & 1,01 & 1,82 & 1,63 \\
G & - & - & - & - & - & - & - & 3,83 & 2,63 & 1,14 & 1,68 \\
H & - & - & - & - & - & - & - & - & 1,23 & 1,67 & 1,40 \\
I & - & - & - & - & - & - & - & - & - & 1,86 & 1,44 \\
J & - & - & - & - & - & - & - & - & - & - & 1,32 \\
\hline
\end{tabular}

Fuente: Elaboración propia. * Nombre de los centros poblados: María Pinto (A), Villa Santa Luisa (B), Santa Emilia (C), La Estrella (D), Chorombo Alto (E), Las Mercedes (F), Baracaldo (G), El Rosario (H), Malalhue (I), Chorombo Bajo (J). 


\subsection{DETERMINACIÓN DE ÁREAS HOMOGÉNEAS DE ACCESIBILIDAD GEOGRÁFICA INTRACOMUNAL}

La determinación de las áreas homogéneas de accesibilidad geográfica intercomunal se realizó considerando como punto de origen (o destino) al centro poblado de María Pinto (A), ya que es el asentamiento que constituye el centro comunal que provee servicios a la población rural circundante, tal como se describió en el método. En la TABLA 8se aprecia una síntesis de la superficie comunal que representa las cuatro categorías de unidades homogéneas mostrando los valores en bruto de las áreas en términos de hectáreas. De este modo nos percatamos que la primera tipología de accesibilidad alta forma un poco más de un tercio. Otro tercio está representado por la categoría de accesibilidad media, lo que permite observar que la comuna presenta disparidades en términos de la accesibilidad intracomunal.

TABLA 8

Superficies totales para las distintas unidades homogéneas de accesibilidad en la comuna de

\begin{tabular}{lrr}
\multicolumn{3}{c}{ María Pinto } \\
\hline Categoría & Superficie (ha) & Proporción (\%) \\
\hline Accesibilidad alta & $13.904,99$ & 35,30 \\
Accesibilidad media & $12.809,69$ & 32,52 \\
Accesibilidad baja & $3.474,51$ & 8,82 \\
Accesibilidad muy baja & $9.032,89$ & 22,93 \\
Total comunal & $39.385,66$ & 100,00 \\
\hline
\end{tabular}

Fuente: Elaboración propia.

La FIGURA 5corresponde a la expresión espacial de la TABLA 8(basada en isócronas), donde además podemos observar los diez centros poblados de la comuna. La primera categoría es la alta accesibilidad la que cubre casi todo el sector del valle, a excepción de algunas estribaciones por la presencia de terrenos en pendiente. Si se compara con la ubicación de los asentamientos, muestra que siete de las diez se encuentran dentro de la categoría. Por supuesto que aquí también está incluido el pueblo de María Pinto (A) como se indicó en el método. El tiempo de desplazamiento de esta tipología es menor a 30 minutos. Por lo tanto, la mayoría de los centros poblados tiene buena accesibilidad a su centro.

La categoría accesibilidad media está distribuida espacialmente alrededor de la categoría anterior representada en parte por las laderas al sur de la comuna y al norte, y más desarrollada al noroeste hacia la cuesta de Ibacache. Los centros poblados que tienen una accesibilidad media (entre 30 y 60 minutos) son Villa Santa Luisa (B) La Estrella (D) y Chorombo Alto (E). La baja accesibilidad hacia el pueblo de María Pinto comprende un tiempo de viaje entre una y dos horas. Su distribución espacial está marcada por ser parte de las laderas hacia el norte y el oeste del territorio y ningún asentamiento de los que fueron analizados se encuentra ubicado dentro de esta. La última categoría es la accesibilidad muy baja, y al igual que en el caso anterior no hay centros poblados localizados en esta tipología. El tiempo de viaje es de más de dos horas y está situado en las altas cumbres de las estribaciones de la cordillera de la Costa y sus abruptas pendientes. 
FIGURA 5

Áreas homogéneas de accesibilidad intracomunal en relación con el centro poblado de la comuna de María Pinto, basadas en isócronas

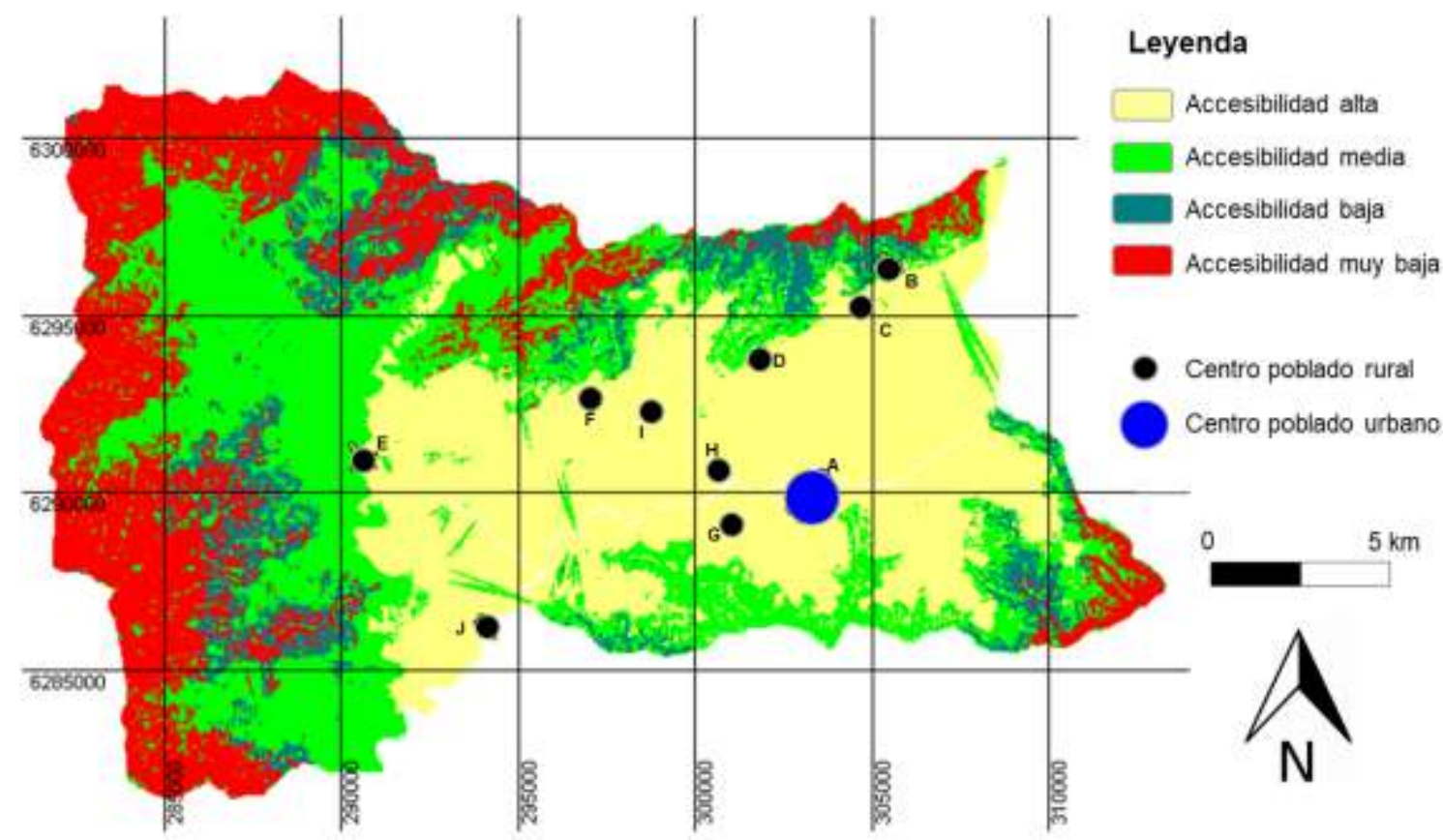

Fuente: Elaboración propia. * Nombre de los centros poblados: María Pinto (A), Villa Santa Luisa (B), Santa Emilia (C), La Estrella (D), Chorombo Alto (E), Las Mercedes (F), Baracaldo (G), El Rosario (H), Malalhue (I), Chorombo Bajo (J).

\section{DISCUSIÓN Y CONCLUSIONES}

\subsection{REFLEXIÓN GENERAL EN TORNO A LOS RESULTADOS}

La presente investigación aporta con la determinación de brechas espaciales en términos de accesibilidad y conectividad geográfica en una comuna de tipo rural del centro de Chile. En este caso de estudio ha sido útil el uso de la aplicación de un modelo matemático que permite comprender el fenómeno espacial de la accesibilidad como lo señala CÉSPEDES ÁLVAREZ (2016). Además, esta investigación aporta con un diagnostico concreto que puede ser utilizado como una herramienta para las políticas de desarrollo y la planificación territorial (PÁEZ et al., 2012; UBILLA BRAVO et al., 2014; UBILLA BRAVO, 2013). Gracias a la reflexión de AUNTA PEÑA (2014), nos interrogamos si María Pinto es un territorio integrado o no. A partir de los resultados obtenidos pensamos que gran parte de la población rural de la comuna está integrada en cuanto a su accesibilidad y conectividad geográfica intracomunal. Esto implica que la población rural tiene un acceso relativamente alto para satisfacer algunas necesidades de servicios que se encuentran en el centro poblado principal. Sin embargo, consideramos que hay otros elementos donde no necesariamente hay una integración. La primera es que hemos considerado los centros asentamientos humanos mayores para el análisis de este estudio. En este sentido se debe considerar otros hábitats como pequeños caseríos que se encuentran en zonas con pendientes más fuertes. Además la cuestión de la integración no sólo apunta a la accesibilidad y a la conectividad geográfica sino también a la percepción de la población respecto de la distancia. Esto lo discutimos más adelante.

\subsection{IMPLICACIONES PRÁCTICAS Y CONTRIBUCIÓN A LA DISCIPLINA}

Desde un punto de vista de la implicación práctica de este artículo, sugerimos seguir avanzando en la disminución de los tiempos de viaje para una mayor accesibilidad 
intracomunal. Tal como señala RUSHTON (1984), hay que avanzar en la mejora de la conectividad geográfica en función de los asentamientos rurales que concentran y que proveen los servicios a la comunidad. En el caso de María Pinto, si bien la mayoría de los asentamientos cuenta con una accesibilidad alta, el Estado debe seguir invirtiendo para cubrir con ese mismo nivel a los centros poblados que no se encuentran en esa categoría sobre todo si se trata de alguna urgencia médica. El fin último del quehacer público es la provisión de condiciones para el bienestar de toda la población.

Junto con ser un aporte al diagnóstico de un territorio rural comunal, este trabajo también contribuye a una corriente de estudios que han analizado estos fenómenos en otro países del sur global (CAROU et al., 2010). Así destacamos varias investigaciones que contribuyeron a la comprensión del fenómeno de la accesibilidad en relación con los servicios de salud rural, entre los que se destacan los casos de comunas o regiones en: México (ANDRADE GARCÍA et al., 2008), Brasil (FERREIRA et al., 2013), Costa Rica (Céspedes Álvarez 2016), Colombia (AUNTA PEÑA, 2014) y Chile (UBILLA BRAVO 2007; SALAZAR et al., 2014; RIFFO ROSAS et al., 2008). Fuera de América Latina destacamos los diagnósticos realizados en Sudáfrica (TANSER, et al., 2006) y República Democrática del Congo(ITONGWA et al., 2002). En particular, apreciamos el trabajo de GÓMEZ FAYRÉN et al., (1990) que ponen acento en la determinación de desequilibrios espaciales relevando la labor de la geografía como disciplina científica.

\subsection{LIMITACIONES Y NUEVAS LÍNEAS DE INVESTIGACIÓN}

Otro punto en discusión trata sobre las limitaciones de esta investigación. Una primera limitante es que trabajamos sobre la base de centros poblados que se encuentran registrados por el INE Chile desde la categoría de caseríos. No obstante, puede haber algunas casas aisladas cuyo tamaño poblacional no fue incluido en la georreferenciación de los datos espaciales. En consecuencia, cuando cruzamos las categorías con los asentamientos, sólo analizamos con aquellos centros que tienen un tamaño de población considerado como caserío, aldea y pueblo, pero puede haber otros casos más pequeños que pueden encontrarse en una categoría de baja o muy baja accesibilidad.

Otra limitación es el uso de algunos indicadores de accesibilidad y conectividad. En este caso, se propone continuar con investigaciones de este tipo que comprenda otros indicadores como los índices alfa, beta y gamma según corresponda (ITONGWA et al., 2002). Estos índices pueden complementar indicando donde efectivamente se puede construir nuevas rutas teniendo presente los circuitos en la red de los caminos de un territorio dado.

Una tercera limitación es que no se mide la satisfacción de la población en torno a la accesibilidad y los servicios básicos. Esto lo indicamos porque en esta investigación nos basamos en las categorías de FARROW et al., (2001) y las adaptamos al medio local. Sin embargo en nuestro estudio no examinamos la satisfacción ni la percepción de accesibilidad alta y/o baja de los propios habitantes ni generamos categorías de accesibilidad dependiendo de la estructura etaria de la población. La opción de este estudio se ha basado en un modelo matemático que nos ayuda a tener una primera visión de estos fenómenos en un medio rural local, pero consideramos que se debe seguir avanzando sobre la percepción de la accesibilidad.

Abordando estas limitaciones, invitamos a continuar este tipo de trabajo en otras áreas rurales de Chile (UBILLA BRAVO, 2007) ya que en general las investigaciones se han ocupado sobre todo en los flujos internos de las grandes áreas urbanas (TRUFFELLO et al., 2015; URETA, 2008). Los investigadores pueden analizar otros casos comunales y también intercomunales con nuevas líneas de investigación incorporando la percepción de la población en el análisis de la accesibilidad y conectividad geográfica. 


\section{BIBLIOGRAFÍA}

ANDRADE GARCÍA, M.D., MACIAS HUERTA, M. del C. y CHÁVEZ HERNÁNDEZ, D. (2008): "Índices de rodeo y accesibilidad a las unidades de atención médica en casos de alacranismo, municipio de Cihuatlán, Jalisco, 2005”. Sincronía, vol. 13, no. 49, pp. 11-30.

AUNTA PEÑA, A.M. (2014):“'Análisis territorial y dinámicas regionales en el noroccidente de Caldas a partir de la conectividad, las centralidades y las capacidades municipales". VI Seminario Internacional de Investigación en Urbanismo, Barcelona-Bogotá. Barcelona, España.

BAVOUX, J.-J., BEAUCIRE, F., CHAPELON, L.y ZEMBRI, P. (2009). Géographie des transports. Paris, France: Armand Colin.

CAROU, H.C. y BRINGEL, B. (2010):“Articulaciones del Sur Global: afinidad cultural, internacionalismo solidario e Iberoamérica en la globalización contrahegemónica”. Geopolítica(s). Revista de estudios sobre espacio y poder, vol. 1, no. 1, pp. 41-63. DOI 10.5209/GEOP.14272.

CÉSPEDES ÁLVAREZ, D.A. (2016):“Diagnóstico de conectividad y accesibilidad de la red vial del cantón de San Carlos, Costa Rica". Caminhos de Geografia, vol. 17, no. 59, pp. 261-278. DOI 10.14393/RCG175915.

DEL CANTO FRESNO, C., GUTIÉRREZ, J. y PÉREZ, M.C. (1998): “Capítulo 4: Asentamientos y transportes". En: C. DEL CANTO FRESNO (ed.), Trabajos prácticos de geografía humana. 2. Madrid, España: Síntesis S.A., Colección Trabajos prácticos de geografía, 2, pp. 201-309.

DOUGLAS, D. H. (1994): "Least-cost Path in GIS Using an Accumulated Cost Surface and Slopelines". Cartographica: The International Journal for Geographic Information and Geovisualization, vol 31, no. 3, pp. 37-51. https://doi.org/10.3138/D327-0323-2JUT-016M.

DUPUY, G. (1985):Systèmes, réseaux et territoires: principes de réseautique territoriale. Paris, France: Presses de l'école nationale des ponts et chaussées.

ESCALONA ORCAO, A.I. y DÍEZ CORNAGO, C.(2003):“Accesibilidad geográfica de la población rural a los servicios básicos de salud: estudio en la provincia de Teruel". Ager. Revista de Estudios sobre Despoblación y Desarrollo Rural, no. 3, pp. 111-149.

ESPINOZA LIZAMA, C. (2010):"La territorialización de la participación ciudadana en el marco de la elaboración de planes de desarrollo comunales". Tiempo y Espacio, vol. 0 , no. 25.

FARROW, A. y NELSON, A. (2001):"Modelación de la Accesibilidad en Arc View 3”. San José, Costa Rica: Centro Internacional de Agricultura Tropical.

FERREIRA, R.V. y DA GRAÇA RAFFO, J. (2012): “O uso dos sistemas de informação geográfica (SIG) no estudo da acessibilidade física aos serviços de saúde pela população rural: revisão da literatura". Hygeia, Revista Brasileira de Geografia Médica e da Saúde, vol. 8, no. 15, pp. 177-189.

FERREIRA, R.V. y DA GRAÇA RAFFO, J. (2013):"Visualização cartográfica da acessibilida de geográfica aos postos de saude da região rural de Registro (SP)". Confins. Revue franco-brésilienne de géographie / Revista franco-brasilera de geografia [en línea], no. 18. [Consulta: 25 junio 2017]. DOI 10.4000/confins.8425. Disponible en: http://confins.revues.org/8425.

GÓMEZ FAYRÉN, J. y LUJÁN MARTÍNEZ, F. (1990):"Estudio de la red de carreteras en la Región de Murcia a través de sus dimensiones básicas". Papeles de Geografía, no. 16, pp. 125-142. 
GRUBESIC, T.H., MATISZIW, T.C., MURRAY, A.T. y SNEDIKER, D.(2008): "Comparative Approaches for Assessing Network Vulnerability”. International Regional Science Review, vol. 31, no. 1, pp. 88-112. DOI 10.1177/0160017607308679.

GUAGLIARDO, M.F.(2004):“Spatial accessibility of primary care: concepts, methods and challenges". International Journal of Health Geographics, vol. 3, pp. 1-13. DOI 10.1186/1476-072X-3-3.

ITONGWA, D.B. y MUNGANGA, J.-P.K.(2002):"Connectivité et accessibilité du réseau de la République Démocratique du Congo". Bulletin de la Société géographique de Liège, no. 42, pp. 61-75.

MONIRUZZAMAN, M., OLARU, D. y BIERMANN, S. (2017):“Assessing the accessibility of activity centres and their prioritisation: a case study for Perth Metropolitan Area". Urban, Planning and Transport Research, vol. 5, no. 1, pp. 121. DOI 10.1080/21650020.2017.1295817.

MORRIS, J.M., DUMBLE, P.L. y WIGAN, M.R.(1979):“Accessibility indicators for transport planning”. Transportation Research Part A: General, vol. 13, no. 2, pp. 91109. DOI 10.1016/0191-2607(79)90012-8.

MUÑIZ SOLARI, O: (1985). Geografía del Transporte y Comunicaciones. Santiago, Chile: Instituto Geográfico Militar.

PÁEZ, A., SCOTT, D.M. y MORENCY, C. (2012):"Measuring accessibility: positive and normative implementations of various accessibility indicators". Journal of Transport Geography, vol. 25, pp. 141-153. DOI 10.1016/j.jtrangeo.2012.03.016.

PIRIE, G.H.(1979):"Measuring Accessibility: A Review and Proposal". Environment and Planning A, vol. 11, no. 3, pp. 299-312. DOI 10.1068/a110299.

RIFFO ROSAS, M., RETAMALES VILLA, C. y MALERMO GUAJARDO, L. (2008): "Condiciones de accesibilidad de los nuevos asentamientos rurales surgidos en el contexto de la modernización productiva en la región del Maule". Investigaciones Geográficas, no. 40, pp. 1-28.

RUSHTON, G.(1984):"Use of Location-Allocation Models for Improving the Geographical Accessibility of Rural Services in Developing Countries". International Regional Science Review, vol. 9, no. 3, pp. 217-240. DOI 10.1177/016001768400900303.

SALAZAR, A., DÍAZ MERY, O., OSSES MC-INTYRE, P. y FOSTER BONNETTE, W.(2014):"Relevancia de la accesibilidad a los centros urbanos, con respecto a la pobreza y ocupación de la población rural y periurbana de la región del Bío-Bío, Chile". Cuadernos de Geografía - Revista Colombiana de Geografía, vol. 23, no. 2, pp. 221-238. DOI 10.15446/rcdg.v23n2.37399.

SEGUÍ PONS, J. M. y PETRUS BEY, J. M. (1991). Geografía de redes y sistemas de transporte. Madrid, España: Editorial Síntesis S.A.

SHIMBEL, A.(1953): "Structural parameters of communication networks". The bulletin of mathematical biophysics, vol. 15, no. 4, pp. 501-507. DOI 10.1007/BF02476438.

TANSER, F., GIJSBERTSEN, B. y HERBST, K.(2006):"Modelling and understanding primary health care accessibility and utilization in rural South Africa: An exploration using a geographical information system". Social Science \& Medicine, vol. 63, no. 3, pp. 691-705. DOI 10.1016/j.socscimed.2006.01.015.

TRUFFELLO, R. y HIDALGO, R.(2015):"Policentrismo en el Área Metropolitana de Santiago de Chile: reestructuración comercial, movilidad y tipificación de subcentros". EURE (Santiago), vol. 41, no. 122, pp. 49-73. DOI 10.4067/S025071612015000100003. 
UBILlA BRAVO, G.(2007):Diagnóstico y Propuesta de Ordenamiento Territorial para la Comuna de Melipilla, Región Metropolitana de Santiago. Memoria para obtención título Geógrafo. Santiago, Chile: Universidad de Chile. DOI 10.13140/2.1.4866.4009.

UBILLA BRAVO, G.(2012):"Entidades rurales aisladas de la Región Metropolitana de Santiago de Chile - RMS: localización y vulnerabilidad”. Cuadernos de Geografía Revista Colombiana de Geografía, vol. 21, no. 2, pp. 127-147.

UBILLA BRAVO, G.(2013):“Análisis de variables clave para el Ordenamiento Territorial. Región Metropolitana de Santiago”. Informe de investigación. Santiago, Chile: Gobierno Regional Metropolitano de Santiago.DOI: 10.13140/RG.2.1.1287.7840.

UBILLA BRAVO, G., HIDALGO VALDIVIA, L. y DÍAZ SCHIFFERLI, H.(2014):Política Pública Regional para el Desarrollo de Localidades Aisladas. Región Metropolitana de Santiago. Santiago, Chile: Gobierno Regional Metropolitano de Santiago. DOI 10.13140/2.1.4776.3848.

URETA, S.(2008):"To Move or Not to Move? Social Exclusion, Accessibility and Daily Mobility among the Low-income Population in Santiago, Chile". Mobilities, vol. 3, no. 2, pp. 269-289. DOI 10.1080/17450100802095338.

WEBER, J.(2006):"Reflections on the future of accessibility". Journal of Transport Geography, vol. 14, no. 5, pp. 399-400. DOI 10.1016/j.jtrangeo.2006.06.005.

YOSHIDA, N. y DEICHMANN, U.(2009):"Measurement of Accessibility and Its Applications". Journal of Infrastructure Development, vol. 1, no. 1, pp. 1-16. DOI 10.1177/097493060900100102. 